\title{
Simulation of Optoelectronic Devices
}

\author{
PAOLO LUGLI*, FABIO COMPAGNONE, ALDO DI CARLO and ANDREA REALE
}

INFM-Dipartimento di Ingegneria Elettronica, Universita di Roma "Tor Vergata”, 00133 Roma, Italy

\begin{abstract}
In the spirit of reviewing various approaches to the modeling and simulation of optoelectronic devices, we discuss two specific examples, related respectively to Semiconductor Optical Amplifiers and to Quantum Cascade Lasers. In the former case, a tight-binding analysis is performed aimed at the optimization of the polarization independence of the device. Further, a rate-equation model is set up to describe the dynamics of gain recovery after optical pumping. A Monte Carlo simulation of a superlattice quantum cascade laser is then presented which provides an insight into the microscopic processes controlling the performance of this device.
\end{abstract}

Keywords: Simulation; Modelling; Photonics; Semiconductor devices; Quantum cascade lasers (QCL); Semiconductor optical amplifiers (SOA)

\section{INTRODUCTION}

Driven mainly by the growing request of fast communication links, the field of optoelectronic device, especially solid-state based, has been witnessing in recent years an increasing attention [1]. One of the crucial issues for optical links is the possibility to amplify the optical power attenuated during the propagation along the fiber without having to resort to an optical-electrical conversion. Therefore, optical amplification of the power transmitted in a fiber link has become a critical point in recent years [2]. Traditionally, optical amplification has been achieved using both semiconductor devices (semiconductor optical amplifiers, SOA) and doped fibers, such as in the erbium doped fiber amplifiers (EDFA). While the latter is by far the most widely used technique at present, SOA's present a series of potential advantages such as integrability, compactness, tunability of the operating wavelength, large gain bandwidth, and non-linear functions (e.g., for use a wavelength converters). Moreover, semiconductor amplifiers can be efficiently used to amplify short optical pulses and can replace other traditional amplifiers used in femtosecond systems. The use of modern advanced growth techniques has lead to the optimization of the performances of such devices thanks to the quantization of the density of states and to band structure engineering. Moreover, the strict control of epitaxial growth permits the

*Corresponding author. 
realization of strained multilayers in an active $M Q W$ region. The introduction of strain in MQWSOAs relates to the need to reduce the polarization dependence of the gain [3].

In the following, we will present a theoretical study of the optical and dynamical properties of SOAs that not only explains the operation of the devices, but opens also the way to its optimization.

In addition to optical communication, several other areas of great economical interest pose an increasing demand for new solid state optical devices. Among them, environmental control and data storage. The latter has pushed researcher towards the development of green-blue lasers based on nitride heterostructures, the former has motivated the interest towards semiconductor infrared and far-infrared (FIR) sources. A description of a modeling approach for nitride-based devices can be found in these Proceedings [4] and will not be treated here. Rather, the numerical simulation of the best solid-state device for the FIR region, namely the Quantum Cascade Laser (QCL), will be discussed in detail. Actually, QCLs are the only valuable semiconductor light source for far-infrared application in the range $4-11 \mu \mathrm{m}$ $[5,6]$.

\section{MODELLING SEMICONDUCTOR OPTICAL AMPLIFIERS}

Optical amplification in SOA's exploits the radiative transitions from states near the top valence band of a direct gap semiconductor to states near the bottom of the conduction band. While SOA's based on bulk materials are already in a very advanced stage, better performance can be achieved via quantum well (QW) structures. Although experimentally such effects are well known and reproducible, a clear theoretical explanation is still missing. The next section describes a tight-binding (TB) approach to the study of the electronic and optical properties of SOAs. In addition to such fundamental issues, the problem of the dynamical non linear response of the SOA to propagating optical pulses is also crucial. The need for useful and precise time domain models of device is twofold. First, one has to check and/or predict performances of real optoelectronic devices in telecommunications applications. On the other side, the investigation of time dependent properties can lead to an understanding of the role played by the various recombination processes taking place inside MQWSOAs [7, 8]. A crucial aspect of this goal is a proper description of the light-carriers interaction. In the second section we will introduce a rate-equation model, and present some comparison with experimental results.

\subsection{A Tight-binding Description of Electronic and Optical Properties}

In QW amplifiers, the increased confinement of carriers and radiation field leads to a significant reduction of threshold currents and power dissipation. Unfortunately, the radiation process supporting optical amplification in QWs results normally in a strong polarization dependence of the amplified signal. In fact, in a typical two dimensional structure the dominant transition occurs between the quantized heavy hole $(\mathrm{HH})$ state and the correspondent conduction band state. Such transition has a strong TE component, which is therefore amplified much more than the TM component related to the light hole ( $\mathrm{LH})$ states. Moreover, the difference between the TE and TM confinement factor enhances the polarization sensitivity of the gain. In order to achieve the desired polarization insensitivity, several SOA structures with strain between the different layers have been proposed [9-11]. The essential feature of the strain is to shift $\mathrm{HH}$ and $\mathrm{LH}$ bands independently. Thus, alignment between the first $\mathrm{HH}$ and the first LH level can be obtained, which results on balanced TM and TE contributions.

Traditionally, in fact, the $\mathbf{k} \cdot \mathbf{p}$ method within the "Effective Mass Approximation" (EFA) is used in the study of two dimensional (2D) structures. However, SOAs have several peculiar characteristics which cannot be investigated in the context 
of EFA. First of all, EFA very poorly reproduces the alignment condition to achieve polarization independence, since it completely fails in describing the $\mathbf{k}_{\|}=0$ mixing when valence band states are resonant in energy [12]. A second characteristic, which is typical of the device we study in this article, consists in the presence of thin $(\simeq 9 \AA)$ $\mathrm{GaAs} \delta$-strained layer. It is well known that EFA cannot be applied to very thin layers [13]. A third point is given by the presence of many different alloys in the same structure. Such make the EFA inapplicable since it is based on the assumption that the periodic part of the Bloch functions do not change between materials composing the structure. While this holds for GaAs/A1GaAs thick quantum structures, it is not clear, up to now, if it can be extended when ultra-thin layers of different materials are used in the same structure.

Due to these limitations, we resort to a more rigorous treatment based on the tight-binding (TB) description of the nanostructure. Details of the method and its application to a variety of optoelectronic and microelectronic devices can be found in Ref. [14, 15], and references therein. Its main advantage is to relax all approximations of EFA without inducing the unpracticable computation cost typical of more ab-initio methods. Optical properties can be easily calculated within the tightbinding scheme without introducing new fitting parameters $[16,17]$.

Strain can be included in the tight-binding model by scaling the hopping matrix elements. The scaling properties of the matrix element has been extensively discussed by Harrison and others $[18,19]$, who showed that an inverse square dependence on the nearest-neighbor distance $(\tau)$ between two atoms reasonably reproduces the chemical properties of several materials, and further improved by several authors [20-22].

In the present work we use the $s p^{3} s^{*}$ tightbinding model [23] with spin - orbit interaction. The numerical implementation of the TB approach is of crucial importance. By itself, the method is computationally quite heavy since the diagonalization of very large matrices is needed. In order to speed up the calculations, we have introduced a hybrid method to diagonalize the tight-binding Hamiltonian which uses a standard (LAPACK [24]) routine to calculate eigenvalues and an inverse iteration scheme [25] to calculate eigenvectors. The advantage of this procedure over other, relies on the fact that only few eigenvectors are needed, namely those closed to the energy band gap.

The $\mathbf{k}_{\|}$integration needed to calculate the absorption/gain coefficient is performed in the 2D Brillouin zone by using a uniform k-points grid in the irreducible wedge, which is obtained by considering the symmetry properties of the Hamiltonian and is defined by the following equations

$$
\begin{aligned}
& 0 \leq k_{x} \leq \frac{1}{2} ; \quad\left|k_{y}\right| \leq k_{x} \\
& \frac{1}{2} \leq k_{x} \leq 1 ; \quad\left|k_{y}\right| \leq 1-k_{x} .
\end{aligned}
$$

Since we are only interested in the absorption/gain coefficient close to the energy gap, we do limit the integration to the region $\left|\mathbf{k}_{\|}\right|<0.12 \pi / a$.

In each optical transition, two nearly spindegenerate valence and conduction subbands are involved. Each squared optical matrix element is summed over the two final conduction states and averaged over the two initial valence states. [12] In our procedure we first calculate (and store) the energy levels and the squared optical matrix elements for each $\mathbf{k}_{\|}$, then we evaluate the absorption/gain coefficient by performing the sums over the carrier distribution functions. To reduce the numerical fluctuations induced by the finite number of $\mathbf{k}_{\|}$points considered in this sum $(\sim 1600)$, we sum over a much finer $\mathbf{k}_{\|}$grid ( $\sim 160000$ points). The energy levels and squared matrix elements at these new $\mathbf{k}_{\|}$points are obtained by using a bilinear interpolation of the calculated quantities. This is allowed since the variation of both energy levels and squared matrix elements in the irreducible wedge are quite smooth.

The reference structure for our study consists of $153 \AA$ wide (52 monolayers) $\operatorname{In}_{0.533} \mathrm{Ga}_{0.467} \mathrm{As}$ 
quantum well surrounded by $\operatorname{In}_{0.74} \mathrm{Ga}_{0.26} \mathrm{As}_{0.56}$ $\mathrm{P}_{0.44}$ barriers, lattice matched to an InP substrate. We investigate the differences in optical matrix elements and gain coefficient between the reference structure and one where 3 monolayers (ML) of InGaAs in the middle of the well are replaced by strained GaAs. The latter system has been shown to guarantee a good degree of polarization insensitivity [11].

The energy band profile of the two systems, i.e., without and with GaAs $\delta$-strain is shown in Figures 1a and $1 \mathrm{~b}$, respectively. The GaAs $\delta$-strain region gives rise to a discontinuity (with respect to InGaAs) $\Delta E_{c}=0.234 \mathrm{eV}$ in the conduction band and $\Delta E_{H H}=-0.267 \mathrm{eV}, \Delta E_{L H}=0.183 \mathrm{eV}$ in the heavy and the light hole bands respectively.

Figures $2 \mathrm{a}$ and $2 \mathrm{~b}$ show the valence band dispersions for the structure without and with GaAs $\delta$-strain, respectively. In these figure the splitting of the spin degenerate bands is not shown since its value is less than $2 \mathrm{meV}$. For the unstrained $\mathrm{QW}$, the first two valence bands have heavy hole character (close to $\mathbf{k}_{\|}=0$ ), while the third one has light hole character. This can be deduced by looking at the squared optical matrix elements, shown in Figure 3a, which are related to the transitions between valence states and the first

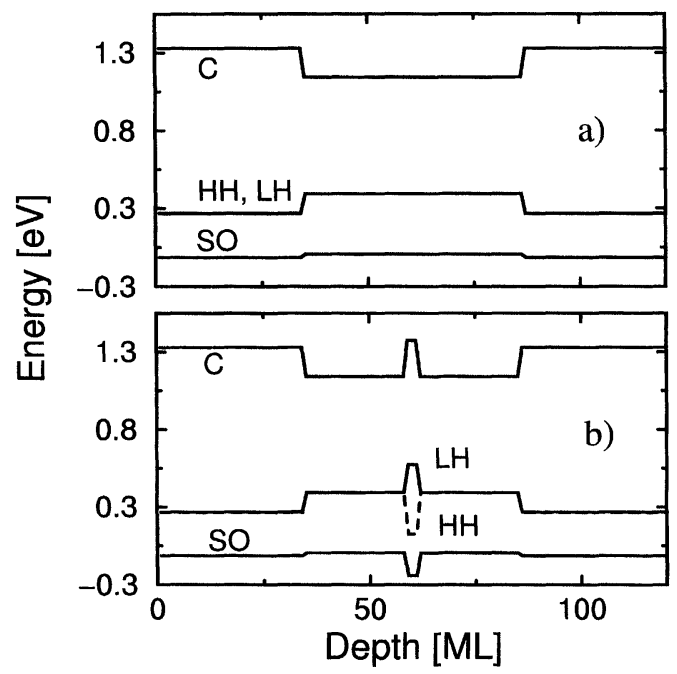

FIGURE 1 Band edge profile for the SOA (a) without and (b) with $\delta$-strain.
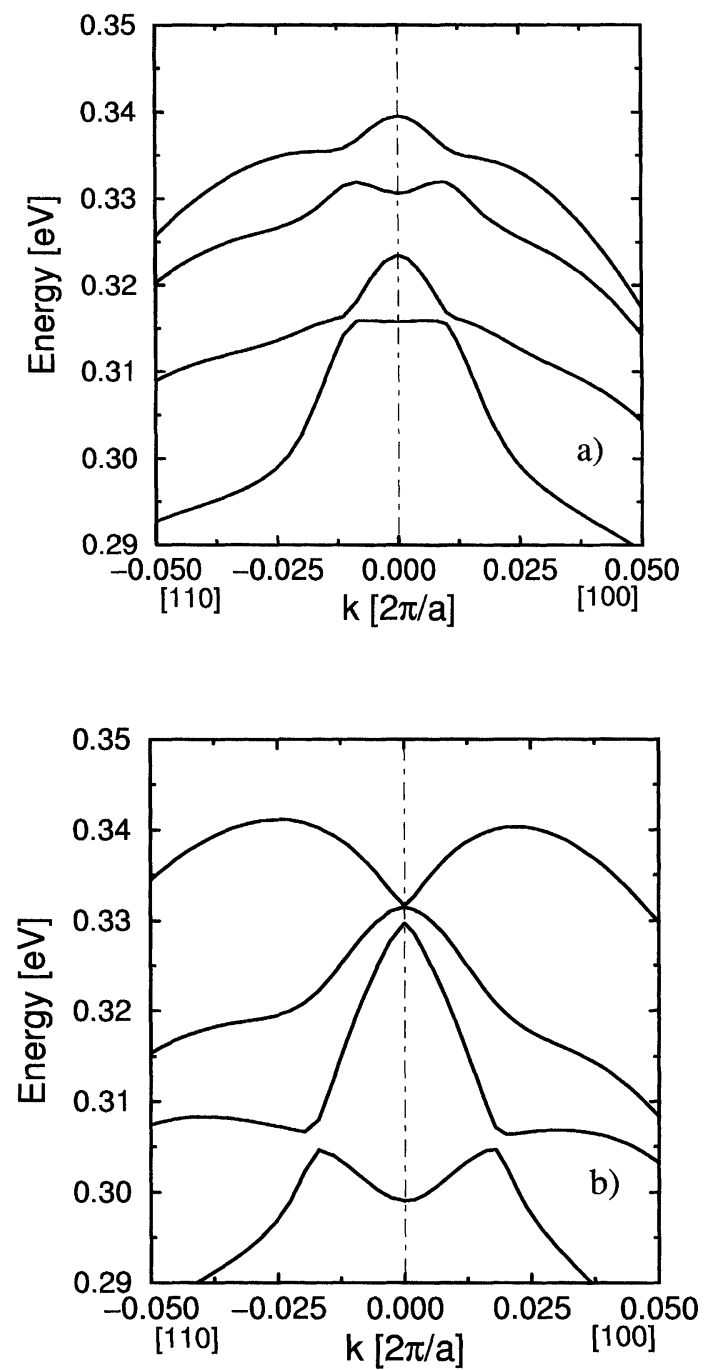

FIGURE 2 Valence band dispersion for the SOA (a) without and (b) with $\delta$-strain.

conduction state. For the transition $V 1 \rightarrow C 1$ (between the first valence band and the first conduction band) the TM contribution vanishes at $\mathbf{k}_{\|}=0$, while the TE contribution dominates. On the other hand, for the $V 3 \rightarrow C 1$ transition the ratio between TM and TE contributions (at $\mathbf{k}_{\|}=0$ ) is close to 4, while the ratio of the TM contribution between this transition and the TE contribution for the $V 1 \rightarrow C 1$ transition is close to $4 / 3$. Such ratios are those typical [26] of a heavy hole (first valence band) and a light hole (third valence 
band) transitions. The second level has a vanishing $\mathrm{TE}$ and $\mathrm{TM}$ contribution for $\mathbf{k}_{\|}=0$. This marks a forbidden transition namely the transition between the second heavy-hole level and the first conduction band level. Moving away from $\mathbf{k}_{\|}=0$, the character of the bands mixes, and both TE and TM contribution are present.

When $\delta$-strain is present the first light-hole level lifts up in energy, as discussed in the previous section, while the first heavy-hole level shifts down, leading to a band degeneration at $\mathbf{k}_{\|}=0$ (see Fig. 2b). The character of these states at zone center can be deduced, as for the case without $\delta$-strain, by looking at the squared optical matrix elements (Fig. 3b). We notice that the first valence band has a light hole character (first LH level), while the second valence band has a heavy-hole character (first $\mathrm{HH}$ level). Very interesting is the third valence band (second $\mathrm{HH}$ band), where the

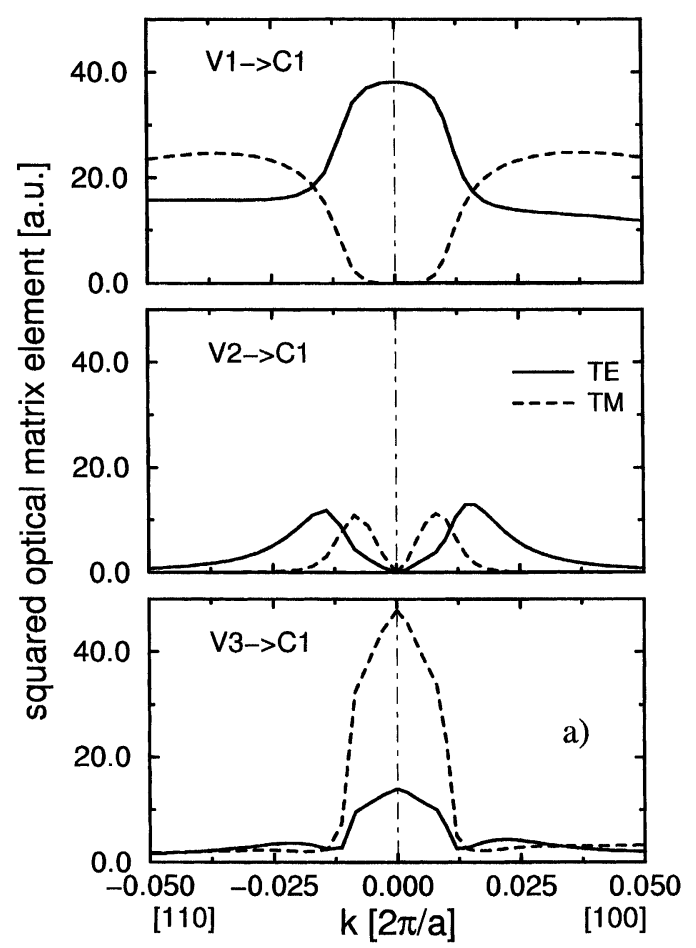

FIGURE 3 Squared optical matrix element as a function of the inplane $k$ vector along [110] and [100] directions for the SOA (a) without and (b) with $\delta$-strain. The contribution of each individual transition is distinguished.

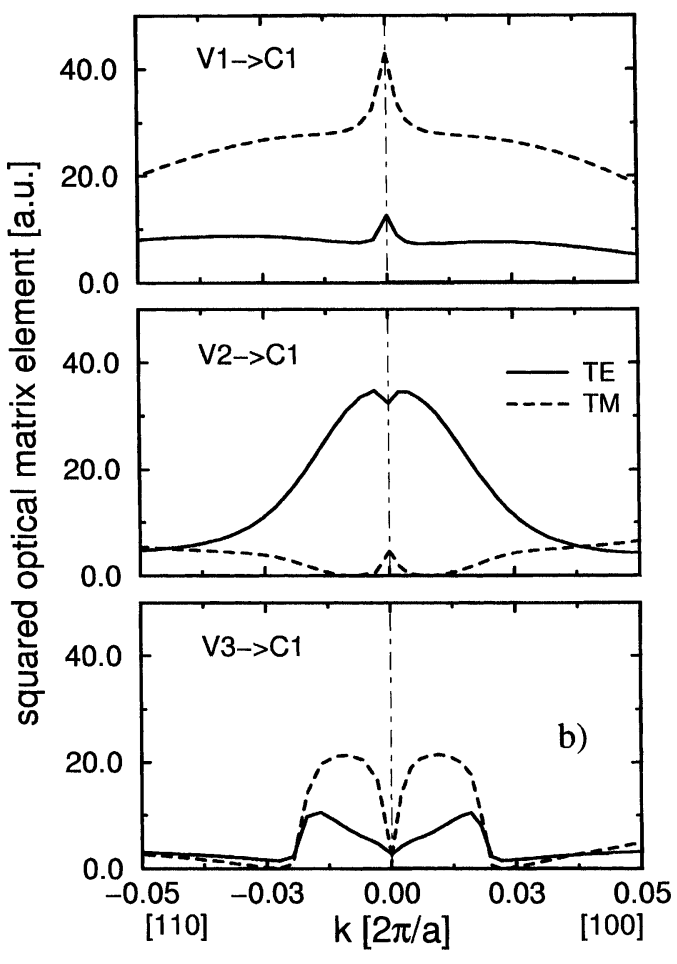

FIGURE 3 (Continued).

transition to $C 1$ presents at $\mathbf{k}_{\|}=0$ a TE contribution. This transition, which cannot be accounted in the $\mathbf{k} \cdot \mathbf{p}$-EFA model, follows from band mixing at $\mathbf{k}_{\|}=0$. The same mixing effect is responsible for the TM polarized $V 2 \rightarrow C 1$ transition at zone center.

The total absorption coefficient for the two systems is shown in Figures $4 \mathrm{a}$ and $4 \mathrm{~b}$. The sharp structures in the absorption coefficient are due to the fact that no broadening has been considering in the calculation. The comparison with experiments would of course require some type of phenomenological broadening of the radiative transition to be considered, which will smoothen the calculated features. The absorption edge for the InGaAs well without $\delta$-strain occurs at $0.774 \mathrm{eV}$. At this energy, only the TE mode is absorbed, since absorption involves the transition between the first valence state, with heavy hole character, and the first conduction band. However, due to the band mixing the TM mode also 

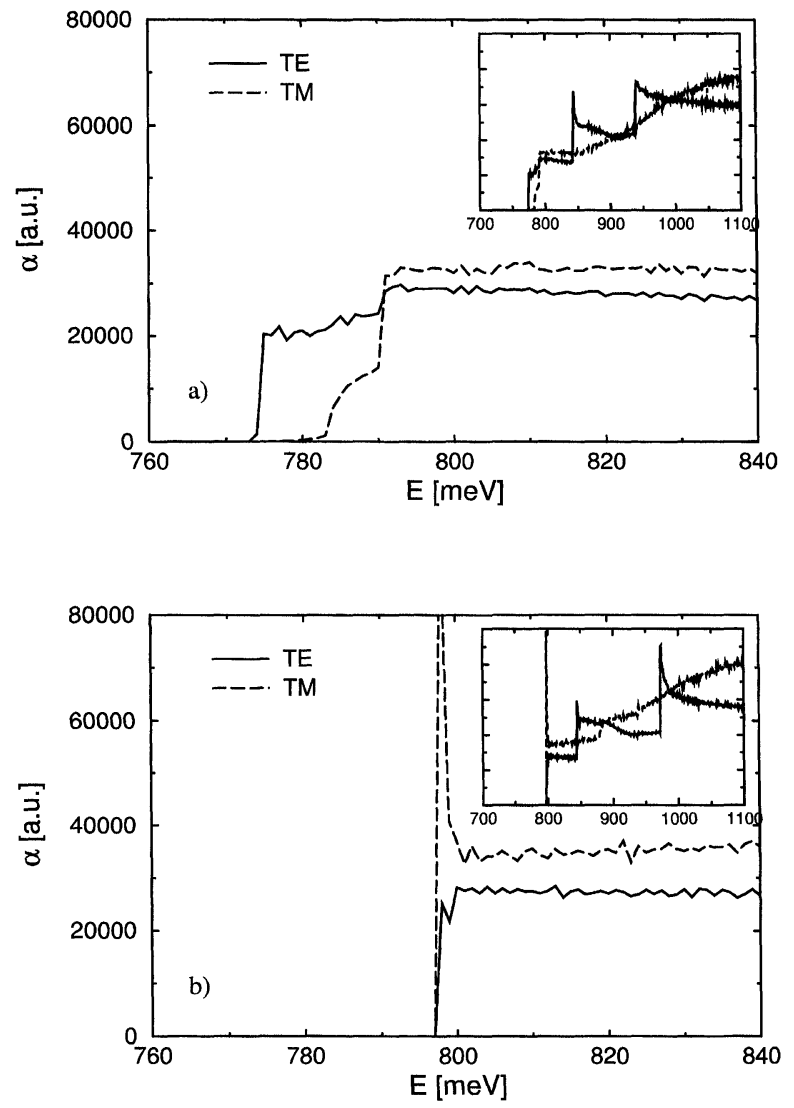

FIGURE 4 Absorption coefficient for the SOA (a) without and (b) with $\delta$-strain. The inserts show an enlarged energy scale. The noise seen in the result is due to the use of a numerical $\mathbf{k}_{\|}$ integration.

begins to be absorbed at slightly higher energies $(0.783 \mathrm{eV})$. Band mixing is also responsible for the contribution to the absorption coefficient from the transition $V 2 \rightarrow C 1$, normally forbidden at $\mathbf{k}_{\|}=0$. For energies higher than $0.790 \mathrm{eV}$, the transition $V 3 \rightarrow C 1$ takes place. Since the $V 3$ band has a light-hole character, the TM mode is now absorbed strongly, at least for $\mathbf{k}_{\|}=0$.

When $\delta$-strain is introduced, the first LH and the first $\mathrm{HH}$ valence states move closer in energy, as can be seen from the absorption coefficient of Figure $4 \mathrm{~b}$. Here the absorption edge, $20 \mathrm{meV}$ higher than without $\delta$-strain is due to the first two valence state ( $\mathrm{LH}$ and $\mathrm{HH}$ character). The peak in the absorption coefficient around the absorption edge deserve some mention. As already observed by Chang and Schulman [12], such a sharp feature is due to negative effective mass of the valence states. Indeed, the first valence state has a negative mass around $\mathbf{k}_{\|}=0$. A more detailed inspection reveals that this mass is similar to the conduction band mass, i.e., $m_{v 1}=0.07 m_{0}$. When the conduction and valence bands are parallel, vertical optical transitions between these bands occur at energy even for $\mathbf{k}_{\|}=0$. Thus several $\mathrm{k}$-points give contributions at the same energy. With respect of Ref. [12], however, the peculiarity in our system consists in the fact that the valence band with negative effective mass corresponds to the first valence band, i.e., the ground state. This implies that such absorption enhancement could be used in a laser structure in order to reduce the current threshold (in a similar way to what is pursued with quantum wire and quantum dots lasers).

Due to the simultaneous transitions from both $\mathrm{LH}$ and $\mathrm{HH}$ states, the final absorption coefficient would tend to be equal for TM and TE modes. However, a difference between the TE and TM polarizations appears in our results (Fig. 4b). Two effects are responsible for such polarization dependence, namely band mixing for $\mathbf{k}_{\|}=0$, and, more important, the presence of the third valence based close in energy to the first two. Such band mixes even at $\mathbf{k}_{\|}=0$ with $\mathrm{HH} 1$ and LH1, thus contributing to the global transition to $C 1$. This effect will not be expected from the simple EFA theory. Actually, the sum of the contributions to the absorption coefficient of the first two valence bands is, indeed, very similar for the TM and the TE. However, the contribution of the third valence band, which occur in the same spectral region, produces the enhancement of the TM absorption.

The above observations lead us to conclude that, in order to have polarization insensitive absorption we need to separate in energy the first two valence band from the rest of the valence bands. Such requirement could indeed be very difficult to fulfill with the $\delta$-strained system, since the perturbation tends to reduce the separation between the 
first two $\mathrm{HH}$ bands. Possible alternatives have been suggested in [15].

In order to calculate the gain coefficient, a proper choice of the quasi-Fermi levels is required. Those are obtained by considering the charge injection from the contacts. We follow a simplified approach where we assume the charge density in the well to be known. The corresponding quasi-Fermi levels are then used to calculate the gain. We assume a room temperature Fermi-Dirac distribution for electron and holes. Many-body effects are not included, however, a generalized Elliot formula can be used to account for such effects [27].

The calculated gain coefficient for the well with and without GaAs $\delta$-strain is shown in Figure $5 \mathrm{~b}$ and Figure $5 \mathrm{a}$, respectively. In both cases we choose a quasi-Fermi level for electron of $150 \mathrm{meV}$
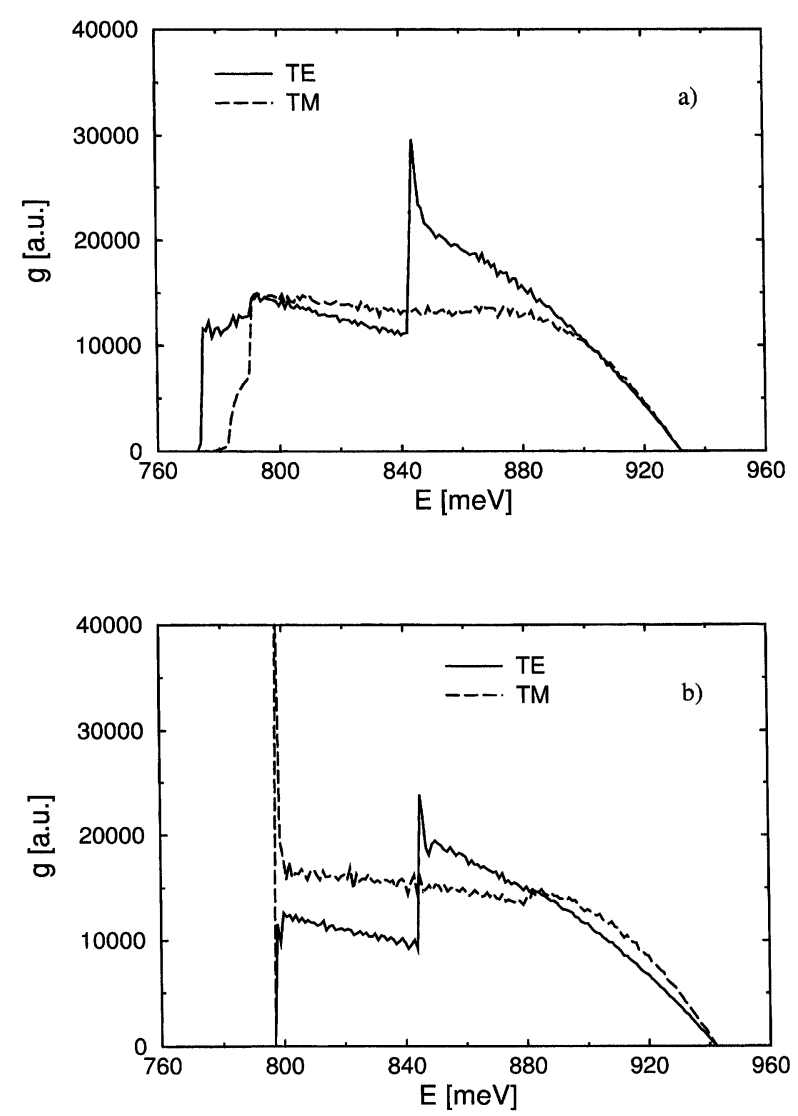

FIGURE 5 Gain coefficient for the SOA (a) without and (b) with $\delta$-strain. from the conduction band edge (see Fig. 1). The quasi-Fermi level of holes is chosen in order to give an equal density of hole with respect to electrons. For the structure without $\delta$-strain we have a hole quasi-Fermi level of $7.88 \mathrm{meV}$ above the top of the valence band, while for the $\delta$-strained structure the hole quasi-Fermi level is $3.28 \mathrm{meV}$ below the top of valence band. Such big difference in the quasi-Fermi level between holes and electron is due to the hole density of the states which is very high compared to the electron one. As for the absorption coefficient, there is a sensible difference for the TE and TM polarization for the system without the $\delta$-strain. The overall difference between TM and TM reduces for the well with $\delta$-strain. However, the presence of the TM spike and the presence of the third valence band make the TM greater than the TE gain close to the band edges. We need to point out, however, that a prevalence of TM contribution to the gain is necessary is SOAs, since it balances the higher confinement factor, of the TE mode with respect to the TM one [28].

\subsection{Rate Question Model for SOAs}

In general, a "rate equation" model is obtained by integrating the current continuity equation, assuming charge neutrality along any device station. Such model has been successfully applied to the description of laser performances [8-29]. For the case of SOAs, the main difference with respect to the laser case is the absence of resonating cavity. We consider here the MQW structure shown in Figure 6 . The structure includes two separate confinement hetero-layers ( $\mathrm{SCH})$ connecting the contact regions to the wells. When coherent phenomena can be neglected (see e.g. [30-31]), the mean electron density $N_{i}$ of each layer $i$ as [32]:

$$
\frac{d N_{i}}{d t}=\eta_{i n j} \frac{I}{q L}-R\left(N_{i}\right)-R_{s t}
$$

where $I$ is the injected current, $\eta_{i n j}$ is the injection efficiency, and $L$ is the thickness of the layer. The 

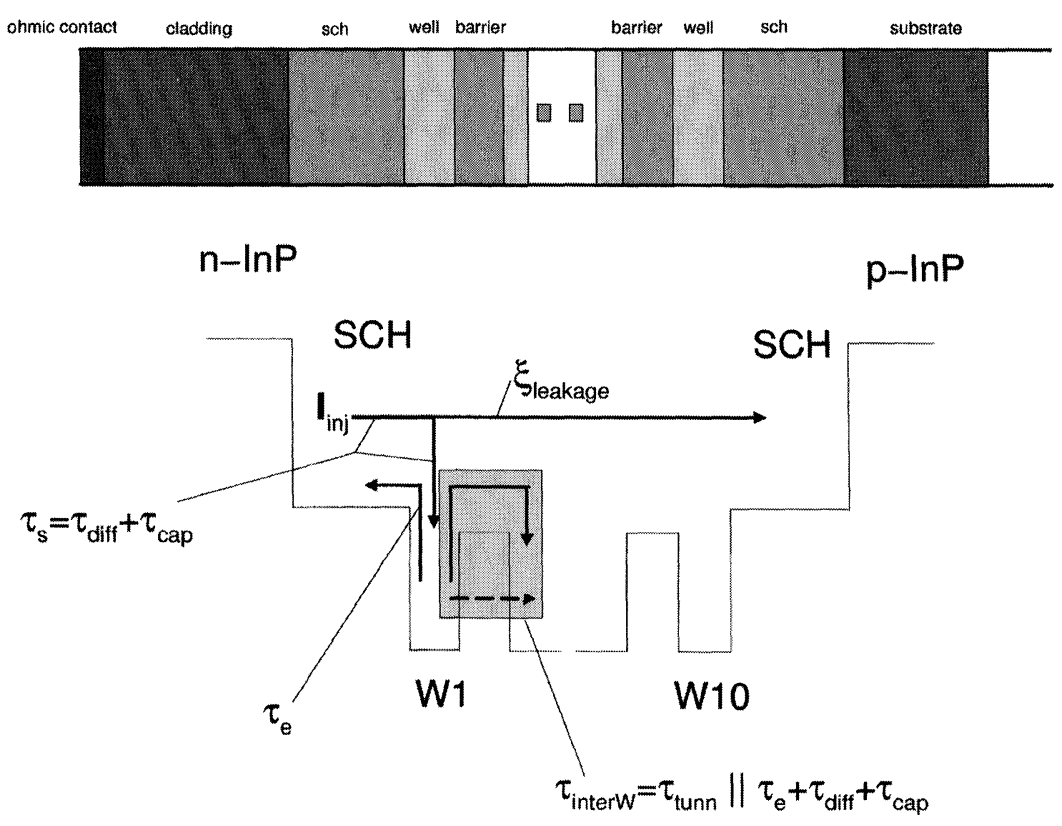

FIGURE 6 Schematic structure of conduction band in a MQW-SOA with more important redistribution mechanisms sketched.

equations are coupled through the parameters that model the carrier transport processes as explained in $[33,34]$.

For the two SCH (labeled 1 and 2 for left and right regions respectively) one can write explicitly:

$$
\begin{gathered}
\frac{d N_{s c h 1}}{d t}=\frac{\eta_{i n j} I}{q L_{s c h}}-\frac{N_{s c h 1}}{\tau_{s}}-\frac{N_{s c h 1}}{\tau_{n s c h}\left(N_{s c h 1}\right)} \\
+\frac{N_{1}}{\tau_{e}} \frac{L_{w e l l}}{L_{s c h}}+\xi \frac{N_{s c h 2}}{\tau_{s}} \\
\frac{d N_{s c h 2}}{d t}=\frac{N_{s c h 2}}{\tau_{s}}-\frac{N_{s c h 2}}{\tau_{n s c h}\left(N_{s c h 2}\right)} \\
+\frac{N_{M}}{\tau_{e}} \frac{L_{w e l l}}{L_{s c h}}+\xi \frac{N_{s c h 1}}{\tau_{s}} .
\end{gathered}
$$

Apart from the injection term appearing in the first equation, the remaining terms account for losses due to diffusive transport and subsequent capture in the adjacent $\mathrm{QW}\left(N_{s c h} / \tau_{s}\right.$, where $\tau_{s}=\tau_{\text {diff }}+$ $\left.\tau_{\text {cap }}\right)$, losses due to non radiative or spontaneous recombination $\left(N_{s c h} / \tau_{s c h}\right.$, where the recombination time $\tau_{\text {sch }}$ depends on carrier density), carrier accumulation due to thermionic emission from the adjacent QW (the total number $\left(N_{q w} \cdot L_{\text {well }}\right) / \tau_{e}$ is normalized with respect to $\mathrm{SCH}$ layer width $L_{s c h}$ ) and exchange term between the two $\mathrm{SCH}$ due to leakage current $\left(\xi\left(N_{s c h} / \tau_{s}\right)\right.$, where $\xi$ is the coupling factor, between 0 and 1$)$.

In MQW systems, one must distinguish between the lateral wells adjacent to the $\mathrm{SCH}$, and inner ones. For the lateral QWs, the following equations hold:

$$
\begin{aligned}
\frac{d N_{1}}{d t}= & (1-\xi) \frac{N_{s c h 1}}{\tau_{s}} \frac{L_{s c h}}{L_{\text {well }}}-\frac{N_{1}}{\tau_{e}}-\frac{N_{1}-N_{2}}{\tau_{c}} \\
& -\frac{N_{1}}{\tau_{n}\left(N_{1}\right)}-M \Gamma_{w} v_{g} g\left(N_{1}\right) S
\end{aligned}
$$

and

$$
\begin{aligned}
\frac{d N_{M}}{d t}= & (1-\xi) \frac{N_{s c h 2}}{\tau_{s}} \frac{L_{s c h}}{L_{\mathrm{well}}}-\frac{N_{M}}{\tau_{e}} \\
& -\frac{N_{M}-N_{M-1}}{\tau_{c}}-\frac{N_{M}}{\tau_{n}\left(N_{M}\right)}-M \Gamma_{w} v_{g} g\left(N_{M}\right) S
\end{aligned}
$$

which include the exchange terms with the $\mathrm{SCH}$ (capture and thermionic emission) and with the 
adjacent QW (inter-well transport). The last term on the right hand side of Eqs. (5) and (6) describes the amplification of the photon density $S$ which experiences a total confinement factor $M \Gamma_{w} \cdot \Gamma_{w}$ and $g(N)$ are, respectively, the confinement factor and gain of each $\mathrm{QW}$.

The density of the central QWs is finally described by:

$$
\begin{aligned}
\frac{d N_{i}}{d t}= & \frac{N_{i-1}-N_{i}}{\tau_{c}}-\frac{N_{i}-N_{i+1}}{\tau_{c}} \\
& -\frac{N_{i}}{\tau_{n}\left(N_{i}\right)}-M \Gamma_{w} v_{g} g\left(N_{i}\right) S .
\end{aligned}
$$

The following formulation, suitable especially for large signal analysis [35], is used for the gain coefficient of the $i$-th QW:

$g\left(N_{i}\right)= \begin{cases}G_{0} \ln \left(\frac{A N_{i}+B N_{i}^{2}+C N_{i}^{3}}{A N_{t h}+B N_{t h}^{2}+C N_{t h}^{3}}\right) ; & N \geq N_{t h} \\ -\alpha ; & N<N_{t h}\end{cases}$

where $\alpha$ is the absorption coefficient [36], and $A N+B N^{2}+C N^{3}$ accounts explicitly for the role of each recombination process through the recombination coefficients $A$ for capture at trap centers, $B$ for spontaneous emission and $C$ for Auger recombination.

The time constant for the recombination mechanisms is given by:

$$
\tau_{n}\left(N_{i}\right)=\frac{1}{A+B N_{i}+C N_{i}^{2}} .
$$

The set of values for the coefficients $A, B$, and $C$ are taken from the literature, and checked versus the comparison with experimental results (see below). The escape of carriers from the QWs is related to thermionic emission over the barriers:

$$
\tau_{e}=\left(\frac{2 \pi m L_{w}^{2}}{k_{B} T}\right)^{1 / 2} \exp \left[\frac{E_{B}}{k_{B} T}\right]
$$

The transport time $\tau_{s}=\tau_{\text {diff }}+\tau_{c a p}$ is modeled considering ambipolar diffusion times in the $\mathrm{SCH}$, while capture time is estimated considering the effect of capture by means of LO phonon emission and also carrier-carrier scattering, which is increasingly important at increasing bias current [37].

The interwell time constant models two concurring processes: tunneling through the barriers on one dies, and thermionic emission plus transport along the barriers with subsequent capture in the adjacent QW on the other (see Fig. 6), i.e.:

$$
\frac{1}{\tau_{c}}=\frac{1}{\left(\tau_{e}+\tau_{d}+\tau_{c}\right)}+\frac{1}{\tau_{t}} .
$$

A list of parameters used in the model is given in Table I.

We want to point out that the definition of the recombination coefficients turns out to be very useful for a compact expression of recombination rates appearings in Eqs. (3) - (7) that two extreme situations are considered for each case, which correspond, respectively, to the minimum and maximum value of the coefficients $\mathrm{A}, \mathrm{B}$ and $\mathrm{C}$ that can be found in the literature for the materials and structure consistent with our sample.

TABLE I Parameter of rate equation model

\begin{tabular}{lcc}
\hline inj. Eff. & $\eta_{\text {inj }}$ & 0.95 \\
width (SCH) & $L_{\text {sch }}$ & $130 \mathrm{~nm}$ \\
width (QW) & $L_{w}$ & $4.8 \mathrm{~nm}$ \\
width (BARR) & $L_{b}$ & $6 \mathrm{~nm}$ \\
barrier heigth (QW-SCH) & $E_{B}$ & $110 \mathrm{meV}$ \\
capture time & $\tau_{c}$ & $1 \mathrm{ps}$ \\
tunnel time & $\tau_{t}$ & $1 \mathrm{ps}$ \\
el mob. (SCH) & $\mu_{n}^{S C H}$ & $0.8 \cdot 10^{4} \mathrm{~cm}^{2} / \mathrm{V} \cdot \mathrm{s}$ \\
holes mob. (SCH) & $\mu_{p}^{S C H}$ & $0.005 \cdot 10^{4} \mathrm{~cm}^{2} / \mathrm{V} \cdot \mathrm{s}$ \\
el mob. (QW) & $\mu_{n}^{S C H}$ & $1.1 \cdot 10^{4} \mathrm{~cm}^{2} / \mathrm{V} \cdot \mathrm{s}$ \\
holes mob. (QW) & $\mu_{p}^{Q W}$ & $0.02 \cdot 10^{4} \mathrm{~cm}^{2} / \mathrm{V} \cdot \mathrm{s}$ \\
temperature & $T$ & $300 \mathrm{~K}$ \\
optical conf. & $\Gamma_{w}$ & 0.02 \\
group vel. & $v_{g}$ & $8.5 \cdot 10^{7} \mathrm{~m} / \mathrm{s}$ \\
Threshold dens. & $N_{t h}$ & $9 \cdot 10^{17} \mathrm{~cm}^{-3}$ \\
min traps coef. & $A_{\min }$ & $10^{8} \mathrm{~s}^{-1}$ \\
min spont. coef f & $B_{\min }$ & $4 \cdot 10^{-11} \mathrm{~cm}^{3} \mathrm{~s}^{-1}$ \\
min auger coef f. & $C_{\min }$ & $5 \cdot 10^{-30} \mathrm{~cm}^{6} \mathrm{~s}-1$ \\
max traps coef f. & $A_{\max }$ & $10^{9} \mathrm{~s}^{-1}$ \\
max spont. coef f & $B_{\max }$ & $2 \cdot 10^{-10} \mathrm{~cm}^{3} \mathrm{~s}^{-1}$ \\
max auger coef f. & $C_{\max }$ & $2 \cdot 10^{-29} \mathrm{~cm}^{6} \mathrm{~s}-1$ \\
initial dens. (SCH) & $N_{s c h 0}$ & $4 \cdot 10^{11} \mathrm{~cm}^{-3}$ \\
initial dens. (QW) & $N_{w 0}$ & $4 \cdot 10^{11} \mathrm{~cm}^{-3}$ \\
leakage factor & $\xi$ & 0.2 \\
\hline & &
\end{tabular}


From Figure 8, we conclude that Auger recombination is the dominant mechanism for the gain compression recovery, thereby being more effective than the Shockley-Read-Hall and

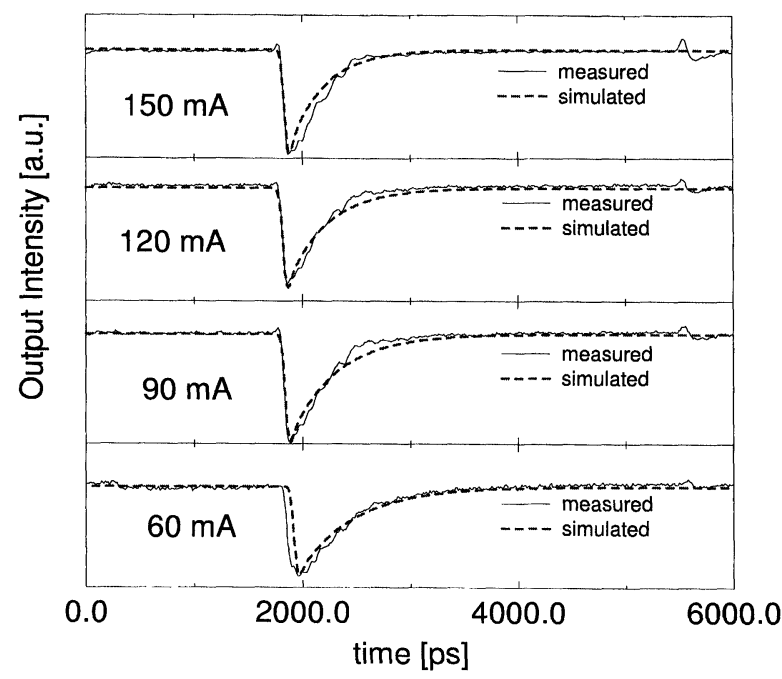

FIGURE 7 Gain compression under high current injection regime. spontaneous recombination processes for all the current (and density) values examined. Indeed, if one considers the case in which all the three processes are considered in the Rate Equation Model, the calculated time constant takes on almost the same values as that for the case of Auger recombination alone. Our results validate simple models (see [8]) which consider only Auger contributions to the gain coefficient.

Different considerations apply to the gain enhancement process observed at low current bias. At currents lower than $40 \mathrm{~mA}$ a sharp peak of duration of less than $100 \mathrm{ps}$ appears in the measured output power (see Fig. 9). In this conditions the SOA is absorbing, and when the pump pulse arrives, it is absorbed. The photogenerated carriers induce population inversion, thereby pushing the SOA above the threshold for gain. After that, the stationary regime is recovered by stimulated recombination, with a characteristic time of tens of picoseconds. Even in this case, theory and experiment agree quite well.
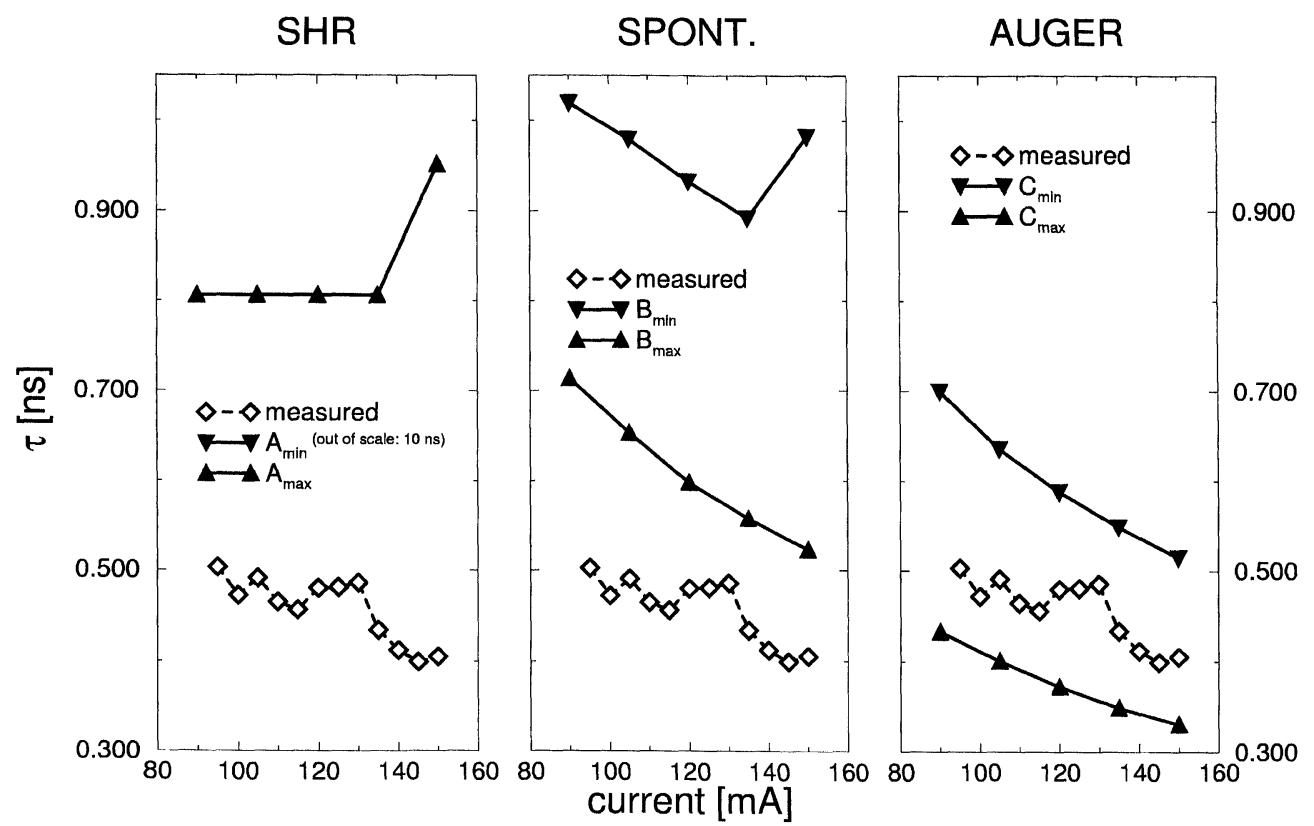

FIGURE 8 Relative importance of the more typical recombination mechanisms on the overall gain compression recovery process in the Rate Equation Model analysis. The time constants extrapolated by the experimental data are also shown. 


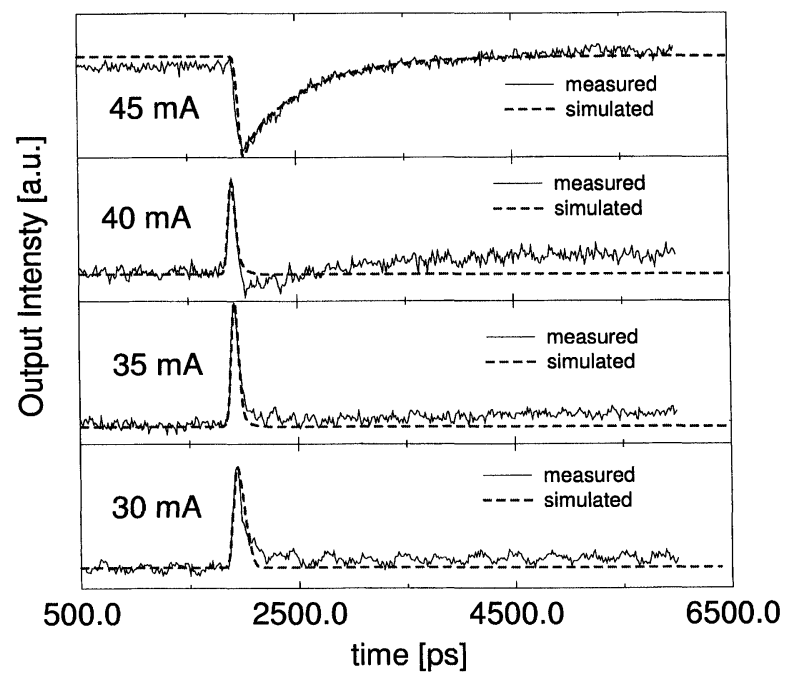

FIGURE 9 Gain dynamics at low injection current regime: from gain compression to gain enhancement.

\section{MONTE CARLO SIMULATION OF SUPERLATTICE QUANTUM CASCADE LASER}

A very interesting realization of QCLs is based on the use of periodic superlattices (SL) as active regions $[6,39]$. This new design concept exhibits new device physics and offers several performance advantages compared with conventional QCL based on coupled quantum wells. So far, the theoretical investigation on the electron dynamics in SL minibands has been very scarce [40]. On the other hand, the modeling of intra-miniband and inter-miniband electronic transitions will eventually lead to a complete understanding of interminiband lasing and further improvements of superlattice QCL devices. In the following we present a theoretical investigation of electron dynamics in $\mathrm{InGaAs} / \mathrm{InA} 1 \mathrm{As}$ superlattice QCL structure $[40,41]$.

For the SL active region of $\mathrm{InGaAs} / \mathrm{InA} 1 \mathrm{As}$ structure $\mathrm{W}=60 \AA \mathrm{B}=18 \AA$ (well and barrier width, respectively), we have calculated the electron energies and wave functions and the scattering rates for electron-phonon interactions [42]. Figure 10 shows the miniband profile and the
electron-LO phonon emission scattering rates, plotted as a function of the electron energy (measured from the bottom of the miniband) at different values of the parallel $k_{p}$ and perpendicular $k_{z}$ k-vector. For instance, for the $2 \rightarrow 2$ transition, the lowest curve corresponds to the maximum $k_{z}$ (at the miniband edge), as only states on the $k_{p}$ dispersion are involved in the transition, while the largest scattering rate is found at $k_{z}=0$, that is at the top of the miniband. The reverse situtaion holds for the intraminiband transitions within the first miniband. A Monte Carlo simulation has been set up based on the scattering rates described above [43]. In addition, electron-electron scattering is included, the interaction being calculated assuming a statically screened Coulomb potential. Pauli exclusion principle is accounted for in the usual way. In order to reach a steady state condition in the MC simulation, electrons are extracted from the bottom of the first miniband at a fixed rate determined by a time constant $\tau_{\text {tunn }}$ and injected in the second miniband at energy $E_{i n j}$, until stationarity is reached.

The stationary distribution functions for the first and second minibands are plotted in the inset of Figure 11. Three different densities are considered. As the density increases, the enhanced intercarrier scattering broadens the distribution
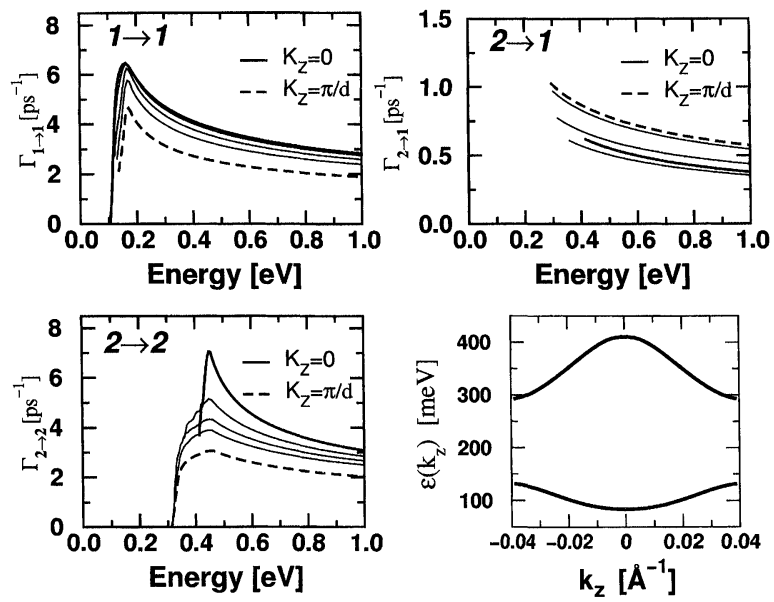

FIGURE 10 Scattering rates and miniband dispersion of the InGaAs/InA1As structure. 


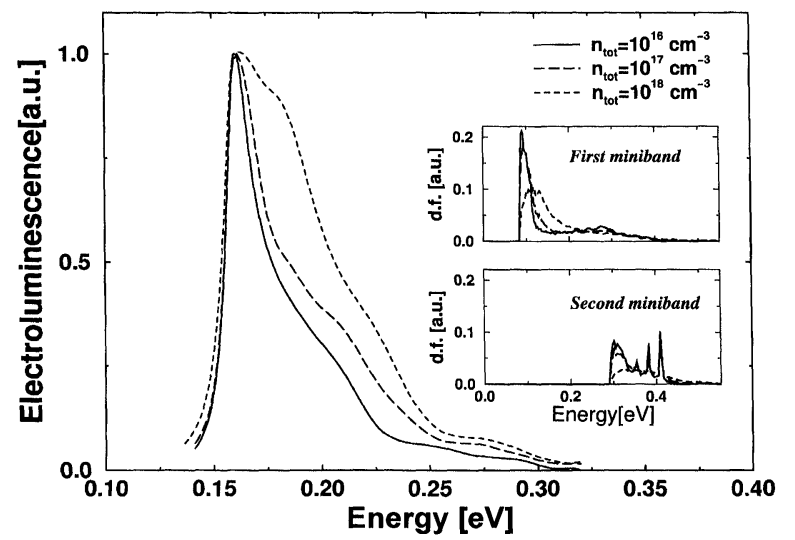

FIGURE 11 Electroluminescence spectra in stationary condition. The inset shows the stationary electron distribution functions of the first and the second minibands.

functions, this reducing the overlap in k-space between the population of the two miniband. The effect of such broadening on the lasing capabilities of the SL can be evaluated by looking at the inversion gain, $g_{i n v}$, which we define as the ratio of the electron density at the bottom of the upper miniband with respect to the density at the top of the lower miniband. The higher the value of $g_{\text {inv }}$, the higher the probability that the structure will lase (and the lower the threshold current for lasing).

An interesting result is found as a function of doping density. As the density moves from $10^{16} \mathrm{~cm}^{-3}$ to $10^{18} \mathrm{~cm}^{-3}$, the inversion gain of the InGaAs/InAlAs structure drops from 26 to 1.2 , indicating that low doping of the active region has to be preferred. The physical reason can be understood from the rates for electron-phonon and electron-electron scattering calculated during the simulation [40]. While the phonon rate does not change appreciably (indicating that Pauli exclusion principle does not effect the electron transport around the miniband edge, at least up to the densities considered here), the intercarrier rate increases with the electron density, both as intra and as inter-miniband process. The former contributes to the thermalization and spreading in $\mathrm{k}$-space of the electrons within each miniband, the latter causes a redistribution between minibands, in particular favoring the scattering out the upper into the lower miniband. Indeed a significant reduction of the threshold current and room temperature operation in QCL with intrinsic SL regions has been reported [44]. The electroluminescence spectra are plotted in Figure 11. The spectra get broader at higher densities, a direct consequence of the electron thermalization detected in the distribution functions. The influence of various simulation parameters has also been tested. In short, we found that the inversion gain is higher when the injection energy is lower. This is caused by a strong reduction of the phonon scattering within the second miniband when electrons are injected closer to the miniband edge, while the interminiband $2 \rightarrow 1$ and the intraminiband $1 \rightarrow 1$ do not show any sizable dependence from $E_{i n j}$. This result indicates the importance of the injection condition in determining the performance of superlattice QCLs. For instance, it is possible to compensate for the loss of inversion gain characteristic of high densities by properly tuning the injection energy. Figure 3 shows the comparison between the electroluminescence spectra of the InGaAs/InA1As SL measured at $5 \mathrm{~K}$ and $300 \mathrm{~K}$. The injected current is $75 \mathrm{~mA}$ in both cases. A detailed description of the experimental

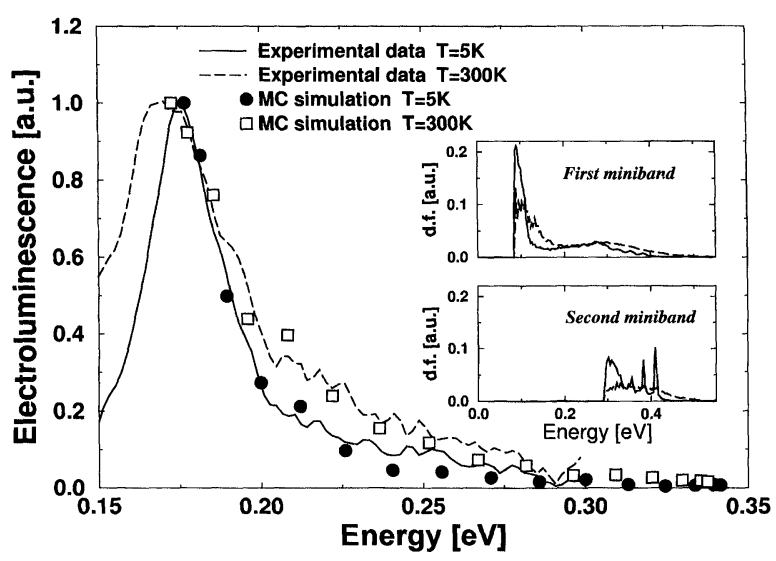

FIGURE 12 Comparison between calculated and measured electroluminescence spectra. The inset shows the electron distribution function at low and room temperature. 
apparatus can be found in Ref. [45]. The main peak is associated with radiative transitions at the minigap between electronic states having wavevectors close to the edge of the SL mini Brillouin zone. The high energy tail corresponds to transitions between states with lower $k_{z}$. The calculated spectra (solid dots in Fig. 12) reproduce well the peak and the high energy tail. The corresponding electron distribution functions are plotted in the inset of Figure 12. We can see that at low temperature the phonon replicas appear clearly in the distributions (but they are washed out in the spectra), and that the population of the edge state is much higher than at room temperature. This is the main reason of the broader spectrum measured at $300 \mathrm{~K}$.

\section{CONCLUSIONS}

We have presented a series of modeling approaches for semiconductor optoelectronic devices, focusing on semiconductor optical amplifiers and quantum cascade lasers. The influence of a delta-strain on the modal absorption/gain characteristic of a semiconductor optical amplifier have been studied by means of a tight-binding calculation. The relation between level alignment, valence band mixing, TE and TM optical transition matrix element and optical absorption/gain has been evidenced via a tight binding approach. The SOA studied under the pump-probe scheme at high injection current regime has also been addressed. The role of the recombination processes have been clarified, from the point of view of gain compression mechanisms exploiting Cross Gain Modulation for WDM applications. We have confirmed that Auger is the most important recombination mechanism for $1.55 \mu \mathrm{m} \mathrm{SOA}$, even if we consider an Auger mechanism reduced in intensity due to valence band modifications induced by strain. Finally a Monte Carlo simulation of the electron dynamics in superlattice QCLs has been presented, which shows the role of phonon and intercarrier scattering processes.

\section{Acknowledgment}

This work was partially supported by INFM under PRA project "SUPERLAS", and by the European TMR Network "Ultrafast Quantum Electronics". We greatfully acknowledge Prof. N. Scamarcio and Dr. D. Campi for the experimental results and for useful discussions.

\section{References}

[1] Sabella, R. and Lugli, P., High Speed Optical Communications, Kluver Academic Publishers, Dordrecht, The Netherland, 1999.

[2] See for example, O'Mahony, M. J., "Optical Amplifiers", in Photonics in Switching, vol. 1, Edited by Midwinter, J. E. Academic Press, San Diego (CA) 1993, and references therein.

[3] Thijs, P. J. A., Tiemeijer, L. F., Binsma, J. J. M. and van Dongen, T. (1994). IEEE Journal of Quantum Electr., 30(2), 477-499.

[4] Sacconi, F., Della Sala, F., Di Carlo, A. and Lugli, P., "Microsocpic modeling of nitride-based heterostructures", these Proceedings.

[5] Faist, J., Capasso, F., Sivco, D. L., Sirtori, C., Hutchinson, A. L. and Cho, A. Y. (1994). Science, 264, 553.

[6] Scamarcio, G., Capasso, F., Sirtori, C., Faist, J., Hutchinson, A. L., Sivco, D. L. and Cho, A. Y. (1997). Science, 276, 773.

[7] Ridley, B. K. (1990). Phys. Rev. B, 41(17), 12190-12196.

[8] Wiesenfeld, J. W., Weiss, S., Botkin, D., Chemla, D. S (1994). Optical and Quantum Electronics, 26(7), S731-S756.

[9] Magari, K., Okamoto, M. and Noguchi, Y. (1991). IEEE Photon. Technol. Lett., 3, 998-1000; Magari, K., Okamoto, M., Suzuki, Y., Sato, K., Noguchi, Y. and Mikami, O. (1994). IEEE J. Quantum Electron., QE-30(3), 695-701.

[10] Debaisieux, G., Guemmouri, M., Chelles, S., Ougazzaden, A., HerveGruyer, G., Filoche, M. and Marzin, J. Y. (1997). IEEE Photon. Technol. Lett., 9(11), 1475-77.

[11] Seiferth, F., Johnson, F. G., Merritt, S. A., Fox, S., Whaley, R. D., Chen, Y. J., Degenais, M. and Stone, D. R. (1997). IEEE Photon. Technol. Lett., 9(10), 1340-1342.

[12] Yia-Chung Chang and Schulman, J. N. (1985). Phys. Rev. $B, 31(4), 2069-2079$.

[13] Zhang, S. B., Yeh, C.-Yu. and Zunger, A. (1993). Phys. Rev. B, 48(15), 11204-11219; Mizel, A. and Cohen, M. L. (1997). Solid State Comm., 104(7), 401-405.

[14] Di Carlo, A. (1998). In: Tight-binding Approach to Computational Materials Science, Mat. Res. Soc. Proc., 491, in press.

[15] Di Carlo, A., Reale, A., Tocca, L. and Lugli, P. (1998). IEEE Journal of Quantum Electr., 34(9), $1730-1739$.

[16] Graf, M. and Vogl, P. (1995). Phys. Rev. B, 51, 4940.

[17] Di Carlo, A., Pescetelli, S., Paciotti, M., Lugli, P. and Graf, M. (1996). Solid State Comm., 98(9), 803-806. 
[18] Harrison, W. A., Electronic Structure and the Properties of Solids, Freeman, San Francisco, CA, 1980.

[19] Majewski, J. A. and Vogl, P. (1989). In: The Structure of Binary Compounds, Edited by de Boer, F. R. and Pettifor, D. G. Elsevier Science Publishers B. V.

[20] Priester, C., Allan, G. and Lannoo, M. (1988). Phys. Rev. B, 37(14), 8519-8519; Phys. Rev. B, 38(14), 9870-9973.

[21] Ma, Q. M., Wang, K. L. and Schulman, J. L. (1993). Phys. Rev. B, 47(4), 1936-1952.

[22] Grosso, G. and Piermarocchi, C. (1995). Phys. Rev. B, 51(23), $16772-16777$.

[23] Vogl, P., Hjalmarson, H. P. and Dow, J. D. (1983). J. Phys. Chem. Solids, 44(5), 365-378.

[24] Anderson, E., Bai, Z., Bischof, C., Demmel, J., Dongarra, J., Du Croz, J., Greenbaum, A., Hammarling, S., McKenney, A., Ostrouchov, S. and Sorensen, D., LAPACK User's Guide, SIAM, Philadelphia, 1992.

[25] Press, W. H., Flannery, B. P., Teukolsky, S. A. and Vetterling, W. T., Numerical recipes, Cambrige University Press, 1986.

[26] Bastard, G., Wave Mechanics Applied to Semiconductor Heterostructure, Les Edition de Physique, Les Ulis Cedex, 1988.

[27] Campi, D. and Coriasso, C. (1995). Phys. Rev. B, 51(16), 10719-10728.

[28] Visser, T. D., Lenstra, D. and Blok, H. (1997). SPIE Proc., 2994, 611-622.

[29] Tessler, N. and Eisenstein, G. (1993). Applied Physics Letters, 62(1), 10-12.

[30] Mark, J. and Mørk, J. (1992). Appl. Phys. Lett., 61(19), $2281-2283$.

[31] Girndt, A., Knorr, A., Hofmann, M. and Koch, S. (1995). Journal of Appl. Phys., 78(5), 2946-2954.
[32] Reale, A., Di Carlo, A., Lugli, P., Campi, D., Cacciatore, C., Stano, A. and Fornuto, G. (1999). IEEE Journal of Quantum Electronics, 35, 1697.

[33] Tessler, N., Nagar, R., Abraham, D. and Eisenstein, G. (1992). Applied Physics Letters, 60(6), 665-667.

[34] Paiella, R., Hunziker, G. and Vahala, K. J. (1996). Applied Physics Letters, 69(27), 4142-4144.

[35] DeTemple, T. A. and Herzinger, C. M. (1993). IEEE Journal of Quantum Electr., 29(5), 1246-1252.

[36] Adachi, S. "Physical Properties of III-V Semiconductor Compounds", J. Wiley \& sons, 1992.

[37] Blom, P. W. M., Haverkort, J. E. M., van Hall, P. J. and Wolter, J. H. (1993). Applied Physics Letters, 62(13), $1490-1492$.

[38] Chick, K. D. (1988). J. Appl. Phys., 63(9), 4688-4698.

[39] For a recent review see Capasso, F., Tredicucci, A., Gmachl, C., Sivco, D. L., Hutchinson, A. L., Cho, A. Y. and Scamarcio, G. (1999). IEEE J. Selected Topics in Quantum Electron, 5, 792.

[40] Tortora, S., Compagnone, F., Di Carlo, A. and Lugli, P. (2000). Physica E7, 20.

[41] Tortora, S., Di Carlo, A. and Paolo Lugli (1999). Physica $B, 272,219$.

[42] Rossi, F., Meier, T., Thomas, P., Koch, S. W., Selbman, P. E. and Molinari, E. (1995). Phys. Rev, B, 51, 16943.

[43] Jacoboni, C. and Lugli, P., "The Monte Carlo Method for Semiconductor Device Simulation", Springer, Wien, 1989.

[44] Tredicucci, A., Capasso, F., Gmachl, C., Sivco, D. L., Hutchinson, A. L., Cho, A. Y., Faist, J. and Scamarcio, G. (1998). Appl. Phys. Lett., 72, 2388.

[45] Scamarcio, G., Capasso, F., Faist, J., Sirtori, C., Sivco, D. L., Hutchinson, A. L. and Cho, A. Y. (1997). Appl. Phys. Lett., 70, 1796. 

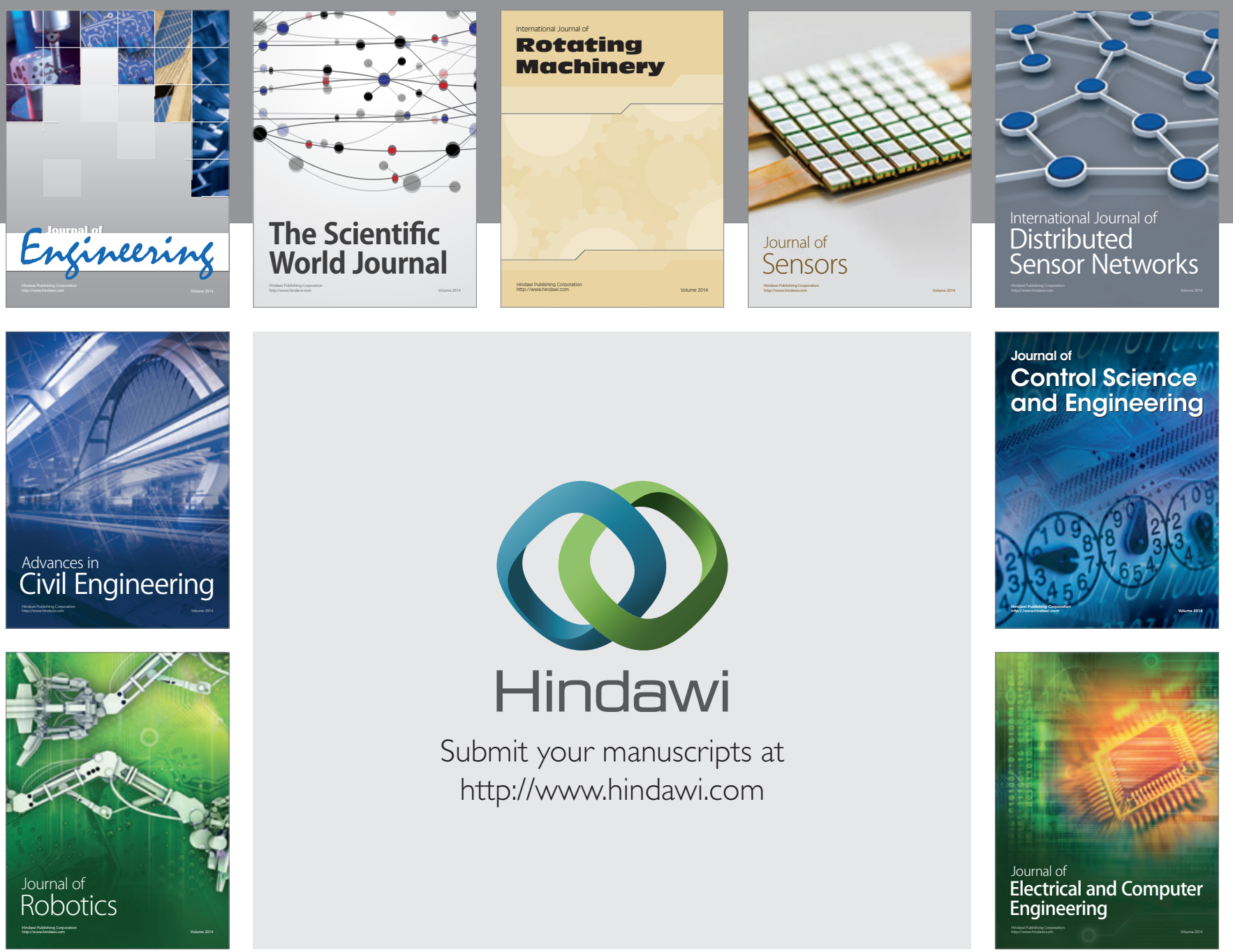

Submit your manuscripts at

http://www.hindawi.com
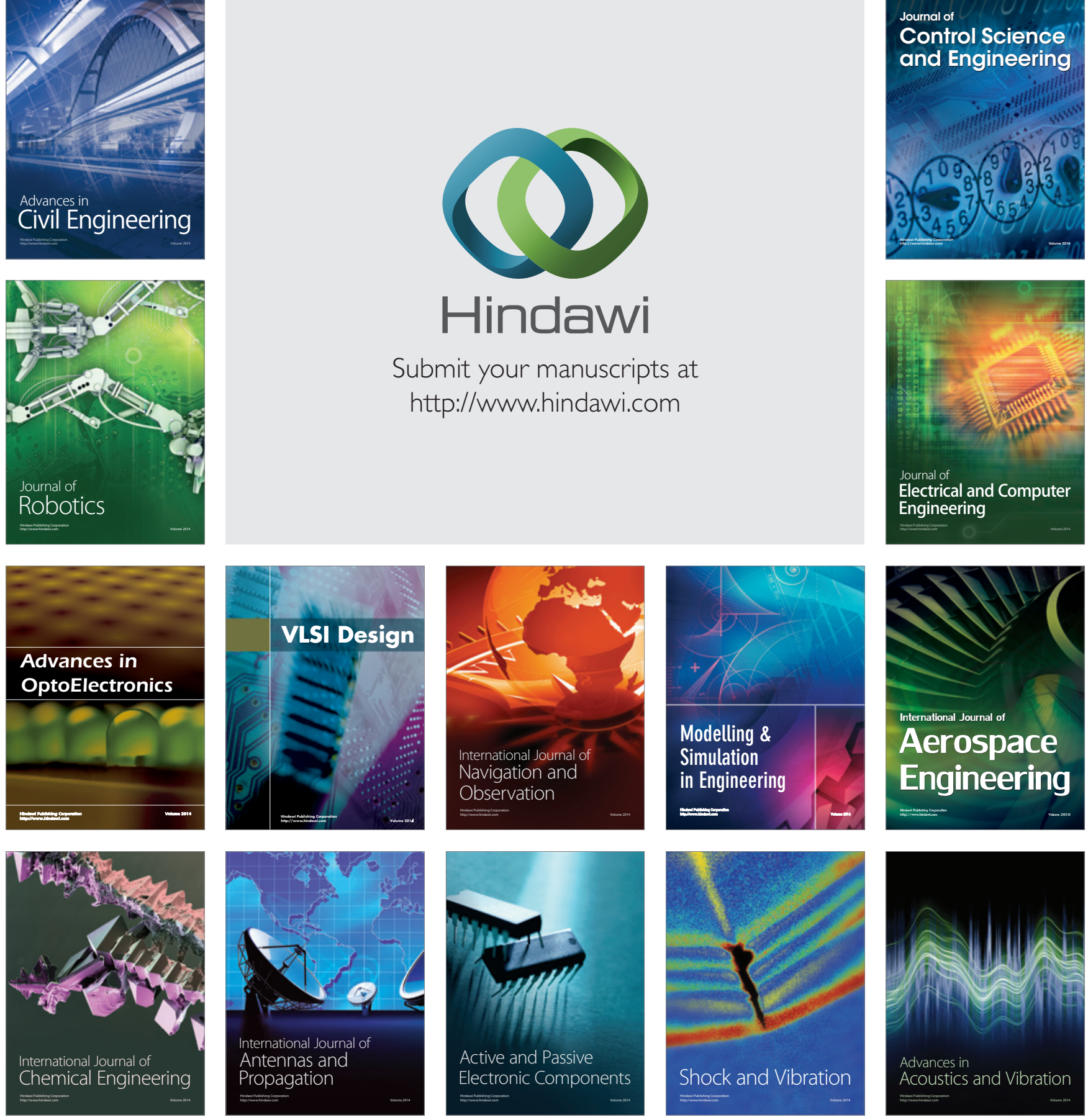